2002 HST Calibration Workshop

Space Telescope Science Institute, 2002

S. Arribas, A. Koekemoer, and B. Whitmore, eds.

\title{
Calibration of Geometric Distortion in the ACS Detectors
}

\author{
G.R. Meurer ${ }^{1}$, D. Lindler ${ }^{2}$, J.P. Blakeslee ${ }^{1}$, C. Cox $^{3}$, A.R. Martel ${ }^{1}$, H.D. Tran ${ }^{1}$, R.J. \\ Bouwens $^{4}$, H.C. Ford ${ }^{1}$, M. Clampin ${ }^{3}$, G.F. Hartig ${ }^{3}$, M. Sirianni ${ }^{1}$, \& G. de Marchi ${ }^{3}$ \\ ${ }^{1}$ Department of Physics and Astronomy, The Johns Hopkins University, \\ Baltimore, MD 21218 \\ 2 Sigma Space Corporation, Lanham, MD 20706 \\ 3 Space Telescope Science institute, Baltimore, MD 21218 \\ ${ }^{4}$ UCO/Lick Observatory, University of California, Santa Cruz, CA 95064
}

\begin{abstract}
The off-axis location of the Advanced Camera for Surveys (ACS) is the chief (but not sole) cause of strong geometric distortion in all detectors: the Wide Field Camera (WFC), High Resolution Camera (HRC), and Solar Blind Camera (SBC). Dithered observations of rich star cluster fields are used to calibrate the distortion. We describe the observations obtained, the algorithms used to perform the calibrations and the accuracy achieved.
\end{abstract}

\section{Introduction}

Images from the Hubble Space Telescope (HST) Advanced Camera for Surveys (ACS) suffer from strong geometric distortion: the square pixels of its detectors project to trapezoids of varying area across the field of view. The tilted focal surface with respect to the chief ray is the primary source of distortion of all three ACS detectors. In addition, The HST Optical Telescope Assembly induces distortion as does the ACS M2 and IM2 mirrors (which are designed to remove HST's spherical aberration). The SBC's optics include a photo-cathode and micro-channel plate which also induce distortion.

Here we describe our method of calibrating the geometric distortion using dithered observations of star clusters. The distortion solutions we derived are given in the IDC tables delivered in Nov 2002, and currently implemented in the STScI CALACS pipeline. This paper is a more up to date summary of our results than that presented at the workshop. An expanded description of our procedures is given by Meurer (2002).

\section{Method}

Observations. The ACS SMOV geometric distortion campaign consisted of two HST observing programs: 9028 which targeted the core of 47 Tucanae (NGC 104) with the WFC and HRC, and 9027 which consisted of SBC observations of NGC 6681. Additional observations from programs 9011, 9018, 9019, 9024 and 9443 were used as additional sources of data, to check the results, and to constrain the absolute pointing of the telescope.

The CCD exposures of 47 Tucanae were designed to well detect stars on the main sequence turn-off at $m_{B}=17.5$ in each frame. This allows for a high density of stars with relatively short exposures. The F475W filter (Sloan g') was used for the CCD observations so as to minimize the number of saturated red giant branch stars in the field. For the HRC two 60s exposures were taken at each pointing, while for the WFC which has a larger time overhead, only one such exposure was obtained per pointing. Simulated images made prior to launch, as well as archival WFPC2 images from Gilliland et al. (2000) were used to check 

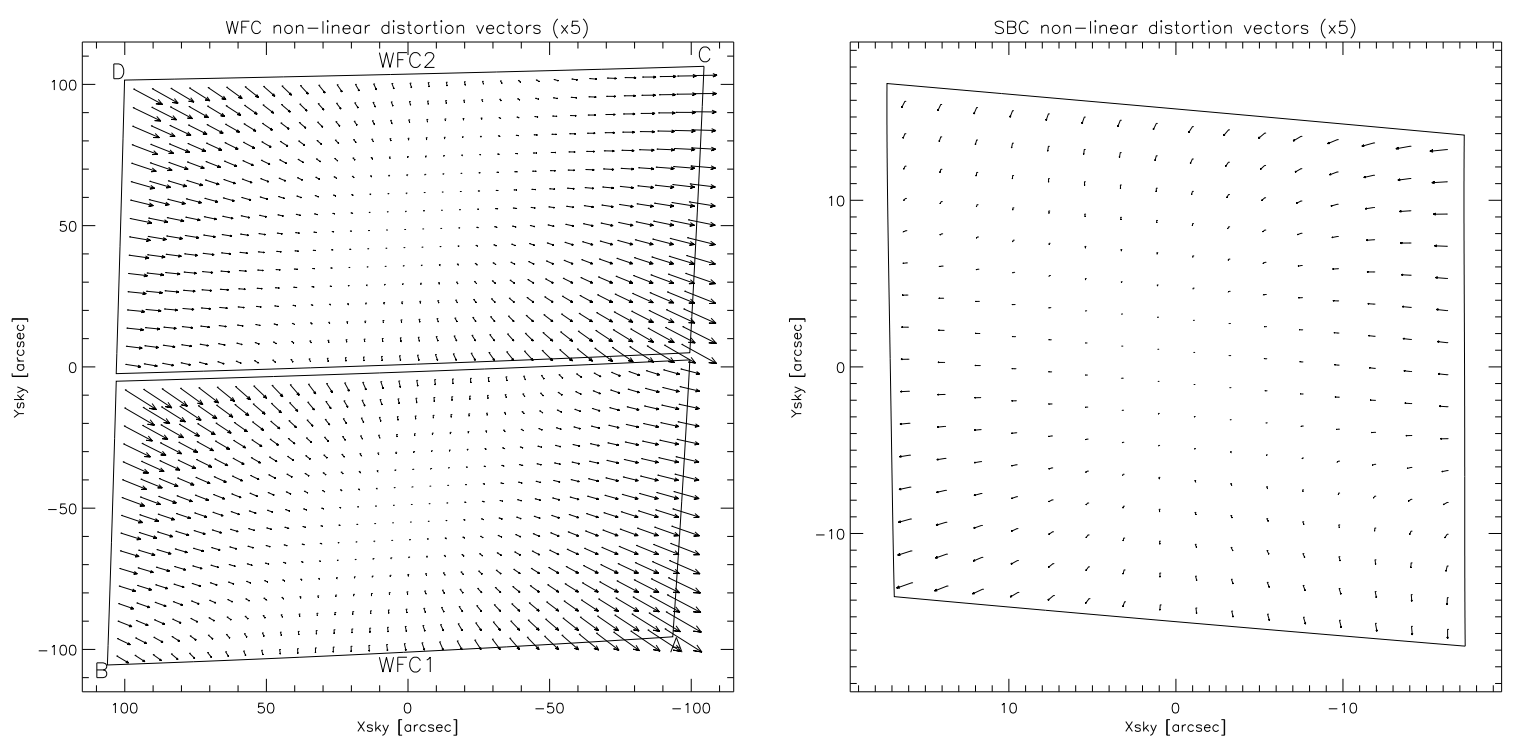

Figure 1. Non linear component to ACS distortion for WFC and SBC detectors.

that crowding would not be an issue. For calibrating the distortion in the SBC we used exposures of NGC 6681 (300s - 450s) which was chosen for the relatively high density of UV emitters (hot horizontal branch stars). The pointing center was dithered around each star field. For the WFC and HRC pointings, the dither pattern was designed so that the offsets between all pairs of images adequately, and non-redundantly, samples all spatial scales from about 5 pixels to $3 / 4$ the detector size. For the SBC pointings, a more regular pattern of offsets is used augmented by a series of 5 pixel offsets.

Distortion model. The heart of the distortion model relates pixel position $(x, y)$ to sky position using a polynomial transformation (Hack \& Cox, 2000) given by:

$$
x_{c}=\sum_{m=0}^{k} \sum_{n=0}^{m} a_{m, n}\left(x-x_{r}\right)^{n}\left(y-y_{r}\right)^{m-n}, \quad y_{c}=\sum_{m=0}^{k} \sum_{n=0}^{m} b_{m, n}\left(x-x_{r}\right)^{n}\left(y-y_{r}\right)^{m-n}
$$

Here $k$ is the order of the fit, $x_{r}, y_{r}$ is the reference pixel, taken to be the center of each detector, or WFC chip, and $x_{c}, y_{c}$ are undistorted image coordinates. The coefficients to the fits, $a_{m, n}$ and $b_{m, n}$, are free parameters. For the WFC, an offset is applied to get the two CCD chips on the same coordinate system:

$$
X^{\prime}=x_{c}+\Delta x(\operatorname{chip} \#), \quad Y^{\prime}=y_{c}+\Delta y(\operatorname{chip} \#) .
$$

$\Delta x$ (chip\#), $\Delta y$ (chip\#) are 0,0 for WFC's chip 1 (as indicated by the FITS CCDCHIP keyword) and correspond to the separation between chips 1 and 2 for chip 2. The chip 2 offsets are free parameters in our fit. $X^{\prime}, Y^{\prime}$ correspond to tangential plane positions in arcseconds which we tie to the HST $V 2, V 3$ coordinate system. Next the positions are corrected for velocity aberration: $X=\gamma X^{\prime}, Y=\gamma Y^{\prime}$, where

$$
\gamma=\frac{1+\mathbf{u} \cdot \mathbf{v} / c}{1-(v / c)^{2}}
$$

Here $\mathbf{u}$ is the unit vector towards the target and $\mathbf{v}$ is the velocity vector of the telescope (heliocentric plus orbital). Neglect of the velocity aberration correction can result in misalignments on order of a pixel for WFC images taken six months apart for targets near 
Table 1. Summary of fit results

\begin{tabular}{lrrccrccc} 
Camera & chip & $\begin{array}{r}\text { pixel } \\
\text { size } \\
\text { [arcsec] }\end{array}$ & Filter & Pointings & $N$ & $\begin{array}{l}\text { rms(x) } \\
\text { [pixels] }\end{array}$ & $\begin{array}{l}\text { rms }(\mathrm{y})^{1} \\
\text { [pixels] }\end{array}$ & Notes \\
\hline WFC & 1 & 0.05 & F475W & 25 & 142289 & 0.042 & 0.045 \\
WFC & 2 & 0.05 & F475W & 25 & 103453 & 0.035 & 0.037 & \\
WFC & 1 & 0.05 & F775W & 10 & 31652 & 0.050 & 0.056 & 2 \\
WFC & 2 & 0.05 & F775W & 10 & 33834 & 0.041 & 0.048 & 2 \\
HRC & & 0.025 & F475W & 20 & 77433 & 0.027 & 0.026 & 3 \\
HRC & & 0.025 & F775W & 13 & 31515 & 0.026 & 0.043 & 3 \\
HRC & & 0.025 & F220W & 12 & 14715 & 0.112 & 0.108 & 3 \\
SBC & & 0.03 & F125LP & 34 & 1561 & 0.109 & 0.094 &
\end{tabular}

\footnotetext{
${ }^{1}$ This is the rms after iteratively clipping measurements with deviations greater than 5 times the rms.

${ }^{2}$ Coefficients held fixed to those found for WFC F475W.

${ }^{3}$ Coefficients held fixed to those found for HRC F475W.
}

the ecliptic. Finally, we must transform all frames to the same coordinate grid on the sky $X_{\text {sky }}, Y_{\text {sky }}$ :

$$
X_{\mathrm{sky}}=\cos \Delta \theta_{i} X-\sin \Delta \theta_{i} Y+\Delta X_{i}, \quad Y_{\mathrm{sky}}=\sin \Delta \theta_{i} X+\cos \Delta \theta_{i} Y+\Delta Y_{i}
$$

where the free parameters $\Delta X_{i}, \Delta Y_{i}, \Delta \theta_{i}$ are the position and rotation offsets of frame $i$.

Calibration algorithm. We use the positions of stars observed multiple times in the dithered star fields to iteratively solve for the free parameters in the distortion solution: fit coefficients $a_{m, n}, b_{m, n}$; chip 2 offsets $\Delta x$ (chip 2), $\Delta y$ (chip 2) (WFC only); frame offsets $\Delta X_{i}, \Delta Y_{i}, \Delta \theta_{i}$; and tangential plane position $X_{\text {sky }}, Y_{\text {sky }}$ of each star used in the fit. The stars are selected by finding local maxima above a selected threshold. The centroid in a $7 \times 7$ box about the local maximum is compared to Gaussian fits to the $x, y$ profiles, if the two estimates of position differ by more than 0.25 pixels, the measurement is rejected as likely being effected by a cosmic ray hit or crowding. Further details of the fit algorithm can be found in Meurer et al. (2002).

Low order terms. Originally only SMOV images taken with a single roll angle were used to define the distortion solutions. The solution using only these data is degenerate in the zeroth (absolute pointing) and linear terms (scale, skewness). So we used the largest commanded offsets with a given guide star pair to set the linear terms. However, comparison of corrected coordinates to astrometric positions showed that residual skewness in the solution remained. Hence, as of November 2002, the IDC tables for WFC and SBC are based on data from multiple roll angles. The overall plate scale is set by the largest commanded offset. For the $\mathrm{HRC}$, the linear scale is set by matching HRC and WFC coordinates, since the same field was used in the SMOV observations. The zeroth order terms (position of the ACS apertures in the HST $V 2, V 3$ frame) was determined from observations of an astrometric field.

\section{Results}

The distortion in all ACS detectors is highly non-linear as illustrated in Fig. 11. We find that a quartic fit $(k=4)$ is adequate for characterizing the distortion to an accuracy much better than our requirement of 0.2 pixels over the entire field of view. Table 1 summarizes the rms of the fits to the various datasets.

The WFC and HRC fits were all to F475W data as noted above. To check the wavelength dependence of the distortion we used data obtained with F775W (WFC and HRC) and F220W (HRC) from programs 9018 and 9019. We held the coefficients fixed and only 

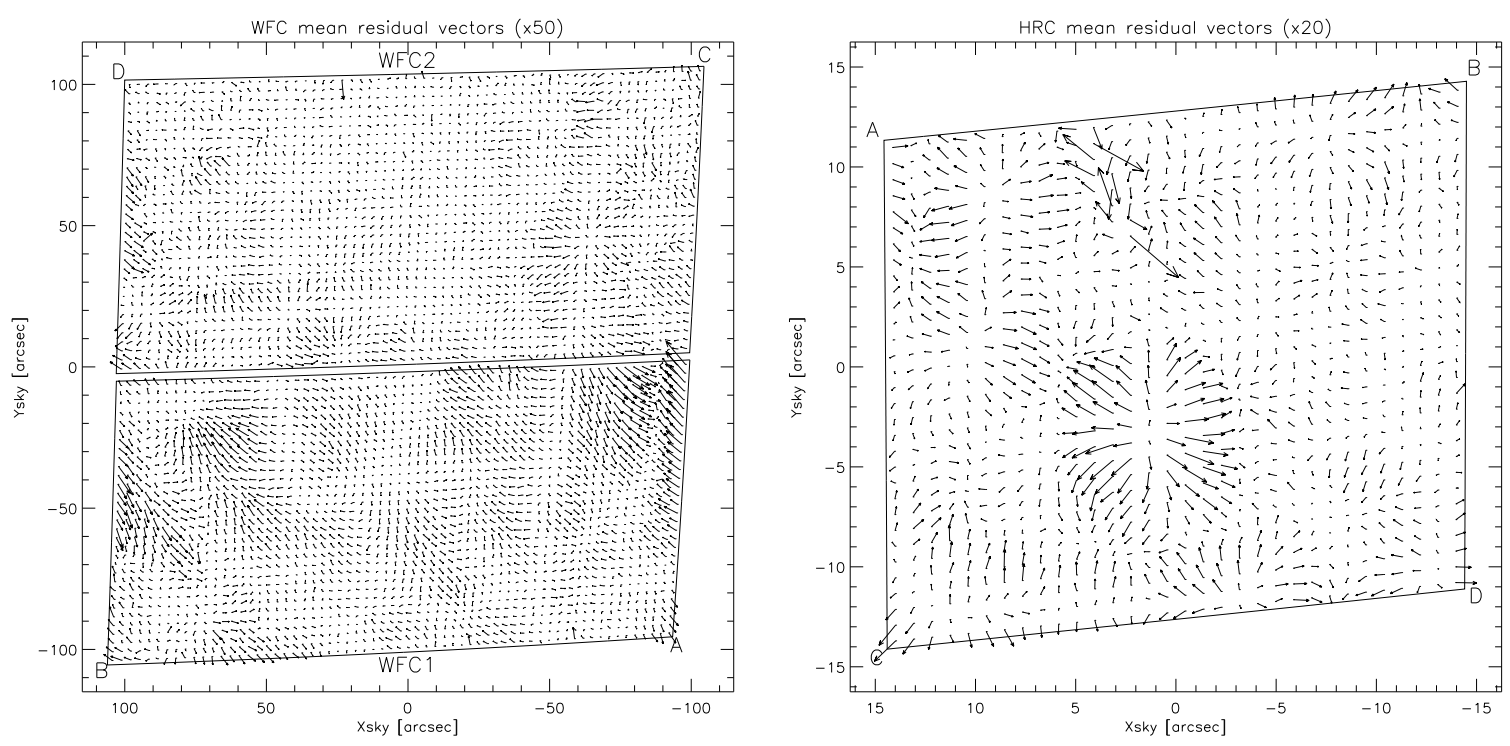

Figure 2. Binned residuals to quartic distortion fits for the WFC and HRC detectors. The large residuals in the HRC map at $X_{\text {sky }} \approx 5^{\prime \prime}, Y_{\text {sky }} \approx 10^{\prime \prime}$ coorespond to the Fastie Finger.

fit the offsets in order to check whether a single distortion solution is sufficient for each detector. Table 2 shows that there is a marginal increase in the rms for the red data of the WFC, little or no increase in the fit rms for the red HRC data, but a significant increase in the rms using the UV data. An examination of the HRC F220W images reveals the most likely cause: the stellar PSF is elongated by 0.1". A similar elongation can also be seen in SBC PSFs. We attribute this to aberration in the optics of either the ACS M1 or M2 mirrors or the HST OTA (Hartig, et al., 2002). The aberration amounts to 0.1 waves at $1600 \AA$, but is negligible relative to optical wavelengths, hence it is not apparent in optical HRC images. While it was expected that the same distortion solution would be applicable to all filters except the polarizers, recent work (by Tom Brown, STScI, and our team) has shown that at least one other optical filter $(\mathrm{F} 814 \mathrm{~W})$ induces a significant plate scale change (factor of $\sim 4 \times 10^{-5}$ ). In the long term, the IDC tables will be selected by filter in the STScI CALACS pipeline.

While a quartic solution is adequate for most purposes, binned residual maps (Fig. 2) show that there are significant coherent residuals in the WFC and HRC solutions. These have amplitudes up to $\sim 0.1$ pixels. The small-scale geometric distortion is the subject of the Anderson \& King contribution to this proceedings.

\section{References}

Hack, W., \& Cox, C. 2000, ISR ACS 2000-11, STScI.

Hartig, G. et al. 2002, in "Future EUV and UV Visible Space Astrophysics Missions and Instrumentation", eds. J.C. Blades \& O.H. Siegmund, Proc. SPIE, Vol. 4854, in press [4854-30].

Gilliland, R.L. et al. 2000, ApJ, 545, L47.

Meurer, G.R. et al. 2002, in "Future EUV and UV Visible Space Astrophysics Missions and Instrumentation", eds. J.C. Blades \& O.H. Siegmund, Proc. SPIE, Vol. 4854, in press [4854-30]. 